\title{
Unsupervised Progressive Learning and the STAM Architecture
}

\author{
James Smith*, Cameron Taylor*, Seth Baer and Constantine Dovrolis ${ }^{\dagger}$ \\ Georgia Institute of Technology \\ \{ jamessealesmith, cameron.taylor, cooperbaer.seth, constantine $\} @$ gatech.edu,
}

\begin{abstract}
We first pose the Unsupervised Progressive Learning (UPL) problem: an online representation learning problem in which the learner observes a nonstationary and unlabeled data stream, learning a growing number of features that persist over time even though the data is not stored or replayed. To solve the UPL problem we propose the Self-Taught Associative Memory (STAM) architecture. Layered hierarchies of STAM modules learn based on a combination of online clustering, novelty detection, forgetting outliers, and storing only prototypical features rather than specific examples. We evaluate STAM representations using clustering and classification tasks. While there are no existing learning scenarios that are directly comparable to UPL, we compare the STAM architecture with two recent continual learning models, Memory Aware Synapses (MAS) and Gradient Episodic Memories (GEM), after adapting them in the UPL setting.
\end{abstract}

\section{Introduction}

The Continual Learning (CL) problem is predominantly addressed in the supervised context with the goal being to learn a sequence of tasks without "catastrophic forgetting" [Goodfellow et al., 2013]. There are several CL variations but a common formulation is that the learner observes a set of examples $\left\{\left(x_{i}, t_{i}, y_{i}\right)\right\}$, where $x_{i}$ is a feature vector, $t_{i}$ is a task identifier, and $y_{i}$ is the target vector associated with $\left(x_{i}, t_{i}\right)$ [Lopez-Paz and Ranzato, 2017]. Other CL variations replace task identifiers with task boundaries that are either given [Hsu et al., 2018] or inferred [Zeno et al., 2018]. Typically, CL requires that the learner either stores and replays some previously seen examples [Rebuffi et al., 2017] or generates examples of earlier learned tasks [Shin et al., 2017].

The Unsupervised Feature (or Representation) Learning $(F L)$ problem, on the other hand, is unsupervised but mostly studied in the offline context: given a set of examples $\left\{x_{i}\right\}$, the goal is to learn a feature vector $h_{i}=f\left(x_{i}\right)$ of a given dimensionality that, ideally, makes it easier to identify the

\footnotetext{
${ }^{*}$ These authors contributed equally to this work.

${ }^{\dagger}$ Contact author
}

explanatory factors of variation behind the data [Bengio et al., 2013], leading to better performance in tasks such as clustering or classification. FL methods differ in the prior $P(h)$ and the loss function. A similar approach is self-supervised methods, which learn representations by optimizing an auxiliary task [Gidaris et al., 2018].

In this work, we focus on a new and pragmatic problem that adopts some elements of CL and FL but is also different than them - we refer to this problem as single-pass unsupervised progressive learning or UPL for short. UPL can be described as follows:

(1) The data is observed as a non-IID stream (e.g., different portions of the stream may follow different distributions and there may be strong temporal correlations between successive examples), (2) the features should be learned exclusively from unlabeled data, (3) each example is "seen" only once and the unlabeled data are not stored for iterative processing, (4) the number of learned features may need to increase over time, in response to new tasks and/or changes in the data distribution, (5) to avoid catastrophic forgetting, previously learned features need to persist over time, even when the corresponding data are no longer observed in the stream.

The UPL problem is encountered in important AI applications, such as a robot learning new visual features as it explores a time-varying environment. Additionally, we argue that UPL is closer to how animals learn, at least in the case of perceptual learning [Goldstone, 1998]. We believe that in order to mimic that, ML methods should be able to learn in a streaming manner and in the absence of supervision. Animals do not "save off" labeled examples to train in parallel with unlabeled data, they do not know how many "classes" exist in their environment, and they do not have to replay/dream periodically all their past experiences to avoid forgetting them.

To address the UPL problem, we describe an architecture referred to as STAM ("Self-Taught Associative Memory")1. STAM learns features through online clustering at a hierarchy of increasing receptive field sizes. We choose online clustering, instead of more complex learning models, because it can be performed through a single pass over the data stream. Further, despite its simplicity, clustering can generate representations that enable better classification performance than more complex FL methods such as sparse-coding or some deep learning

\footnotetext{
${ }^{1}$ Code available at https://github.com/CameronTaylorFL/stam
} 


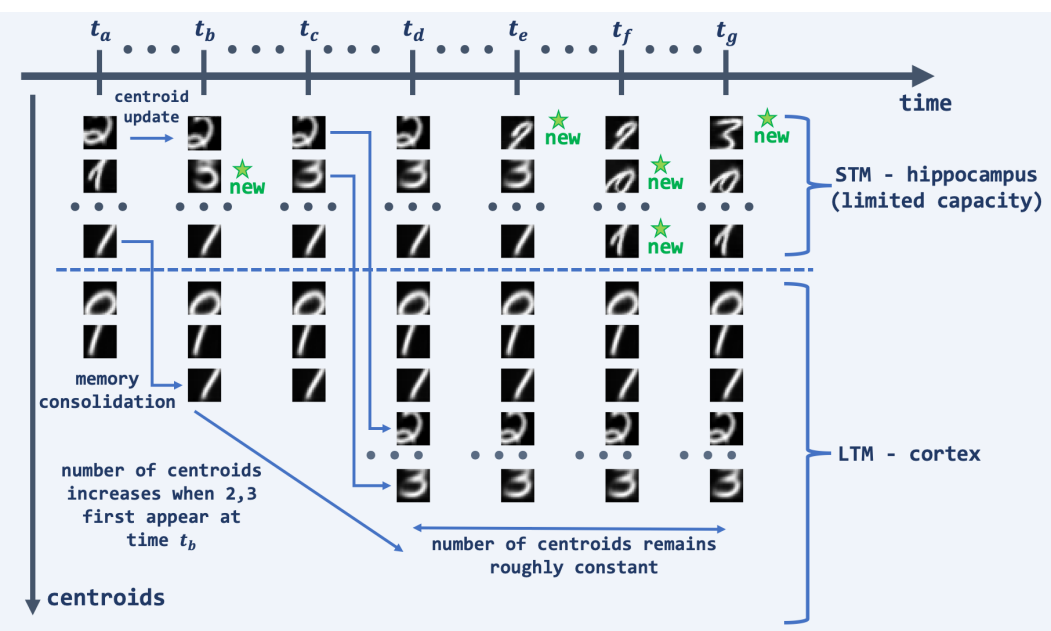

Figure 1: A hypothetical pool of STM and LTM centroids visualized at seven time instants. From $t_{a}$ to $t_{b}$, a centroid is moved from STM to LTM after it has been selected $\theta$ times. At time $t_{b}$, unlabeled examples from classes ' 2 ' and ' 3 ' first appear, triggering novelty detection and new centroids are created in STM. These centroids are moved into LTM by $t_{d}$. From $t_{d}$ to $t_{g}$, the pool of LTM centroids remains the same because no new classes are seen. The pool of STM centroids keeps changing when we receive "outlier" inputs of previously seen classes. Those centroids are later replaced (Least-Recently-Used policy) due to the limited capacity of the STM pool.

methods [Coates et al., 2011]. STAM allows the number of clusters to increase over time, driven by a novelty detection mechanism. Additionally, STAM includes a brain-inspired dual-memory hierarchy (short-term versus long-term) that enables the conservation of previously learned features (to avoid catastrophic forgetting) that have been seen multiple times at the data stream, while forgetting outliers. To the extent of our knowledge, the UPL problem has not been addressed before. The closest prior work is CURL ("Continual Unsupervised Representation Learning") [Rao et al., 2019]. CURL however does not consider the single-pass, online learning requirement. We further discuss this difference with CURL in Section 7.

\section{STAM Architecture}

In the following, we describe the STAM architecture as a sequence of its major components: a hierarchy of increasing receptive fields, online clustering (centroid learning), novelty detection, and a dual-memory hierarchy that stores prototypical features rather than specific examples. The notation is summarized for convenience in the Supplementary Material (SM) [Smith et al., 2021].

I. Hierarchy of increasing receptive fields: An input vector $\mathbf{x}_{\mathbf{t}} \in \mathbb{R}^{\mathbf{n}}$ (an image in all subsequent examples) is analyzed through a hierarchy of $\Lambda$ layers. Instead of neurons or hiddenlayer units, each layer consists of STAM units - in its simplest form a STAM unit functions as an online clustering module. Each STAM unit processes one $\rho_{l} \times \rho_{l}$ patch (e.g. $8 \times 8$ subvector) of the input at the $l$ 'th layer. The patches are overlapping, with a small stride (set to one pixel in our experiments) to accomplish translation invariance (similar to CNNs). The patch dimension $\rho_{l}$ increases in higher layers - the idea is that the first layer learns the smallest and most elementary features while the top layer learns the largest and most complex features.

II. Centroid Learning: Every patch of each layer is clustered, in an online manner, to a set of centroids. These time-varying centroids form the features that the STAM architecture gradually learns at that layer. All STAM units of layer $l$ share the same set of centroids $C_{l}(t)$ at time $t$ - again for translation invariance. ${ }^{2}$ Given the $m$ 'th input patch $\mathbf{x}_{\mathbf{l}, \mathbf{m}}$ at layer $l$, the nearest centroid of $C_{l}$ selected for $\mathrm{x}_{1, \mathrm{~m}}$ is

$$
\mathbf{c}_{\mathbf{l . j}}=\arg \min _{c \in C_{l}} d\left(\mathbf{x}_{\mathbf{l}, \mathbf{m}}, \mathbf{c}\right)
$$

where $d\left(\mathbf{x}_{1, \mathbf{m}}, \mathbf{c}\right)$ is the Euclidean distance between the patch $\mathbf{x}_{\mathbf{l}, \mathrm{m}}$ and centroid $\mathbf{c} .^{3}$ The selected centroid is updated based on a learning rate parameter $\alpha$, as follows:

$$
\mathbf{c}_{\mathbf{l}, \mathbf{j}}=\alpha \mathbf{x}_{\mathbf{l}, \mathbf{m}}+(1-\alpha) \mathbf{c}_{\mathbf{l , j}}, \quad 0<\alpha<1
$$

A higher $\alpha$ value makes the learning process faster but less predictable. A centroid is only updated by at most one patch and the update is not performed if patch is considered "novel" (defined in the next paragraph). We do not use a decreasing value of $\alpha$ because the goal is to keep learning in a non-stationary environment rather than convergence to a stable centroid.

III. Novelty detection: When an input patch $\mathrm{x}_{\mathbf{1}, \mathrm{m}}$ at layer $l$ is significantly different than all centroids at that layer (i.e., its distance to the nearest centroid is a statistical outlier), a new centroid is created in $C_{l}$ based on $\mathbf{x}_{1, \mathbf{m}}$. We refer to this event as Novelty Detection (ND). This function is necessary so that the architecture can learn novel features when the data distribution changes. To do so, we estimate in an online manner the distance distribution between input patches and their nearest centroid (separately for each layer). The novelty detection threshold at layer $l$ is denoted by $\hat{D}_{l}$ and it is defined as the 95 -th percentile $(\beta=0.95)$ of this distance distribution.

\footnotetext{
${ }^{2}$ We drop the time index $t$ from this point on but it is still implied that the centroids are dynamically learned over time.

${ }^{3}$ We have also experimented with the L1 metric with only minimal differences. Different distance metrics may be more appropriate for other types of data.
} 


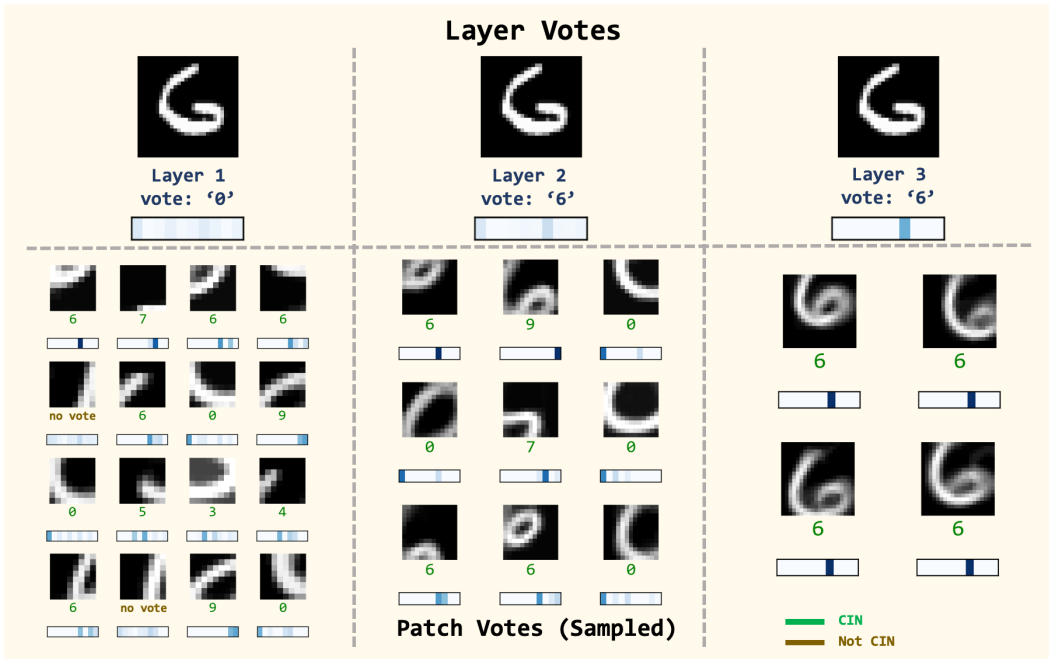

Figure 2: An example of the classification process. Every patch (at any layer) that selects a CIN centroid votes for the single class that has the highest association with. These patch votes are first averaged at each layer. The final inference is the class with the highest cumulative vote across all layers.

IV. Dual-memory organization: New centroids are stored temporarily in a Short-Term Memory (STM) of limited capacity $\Delta$, separately for each layer. Every time a centroid is selected as the nearest neighbor of an input patch, it is updated based on (2). If an STM centroid $\mathbf{c}_{1, \mathrm{j}}$ is selected more than $\theta$ times, it is copied to the Long-Term Memory (LTM) for that layer. We refer to this event as memory consolidation. The LTM has (practically) unlimited capacity and a much smaller learning rate (in our experiments the LTM learning rate is set to zero).

This memory organization is inspired by the Complementary Learning Systems framework [Kumaran et al., 2016], where the STM role is played by the hippocampus and the LTM role by the cortex. This dual-memory scheme is necessary to distinguish between infrequently seen patterns that can be forgotten ("outliers"), and new patterns that are frequently seen after they first appear ("novelty").

When the STM pool of centroids at a layer is full, the introduction of a new centroid (created through novelty detection) causes the removal of an earlier centroid. We use the LeastRecently Used (LRU) policy to remove atypical centroids that have not been recently selected by any input. Figure 1 illustrates this dual-memory organization.

V. Initialization: We initialize the pool of STM centroids at each layer using randomly sampled patches from the first few images of the unlabeled stream. The initial value of the novelty-detection threshold is calculated based on the distance distribution between each of these initial STM centroids and its nearest centroid.

\section{Clustering Using STAM}

We can use the STAM features in unsupervised tasks, such as offline clustering. For each patch of input $\mathbf{x}$, we compute the nearest LTM centroid. The set of all such centroids, across all patches of $\mathbf{x}$, is denoted by $\Phi(\mathbf{x})$. Given two inputs $\mathbf{x}$ and $\mathbf{y}$, their pairwise distance is the Jaccard distance of $\Phi(\mathbf{x})$ and $\Phi(\mathbf{y})$. Then, given a set of inputs that need to be clustered, and a target number of clusters, we apply a spectral clustering algorithm on the pairwise distances between the set of inputs. We could also use other clustering algorithms, as long as they do not require Euclidean distances.

\section{Classification Using STAM}

Given a small amount of labeled data, STAM representations can also be evaluated with classification tasks. We emphasize that the labeled data is not used for representation learning it is only used to associate previously learned features with a given set of classes.

I. Associating centroids with classes: Suppose we are given some labeled examples $X_{L}(t)$ from a set of classes $L(t)$ at time $t$. We can use these labeled examples to associate existing LTM centroids at time $t$ (learned strictly from unlabeled data) with the set of classes in $L(t)$.

Given a labeled example of class $k$, suppose that there is a patch $\mathrm{x}$ in that example for which the nearest centroid is c. That patch contributes the following association between centroid $\mathbf{c}$ and class $k$ :

$$
f_{\mathbf{x}, \mathbf{c}}(k)=e^{-d(\mathbf{x}, \mathbf{c}) / \bar{D}_{l}}
$$

where $\bar{D}_{l}$ is a normalization constant (calculated as the average distance between input patches and centroids). The classassociation vector $\mathrm{g}_{\mathbf{c}}$ between centroid $\mathbf{c}$ and any class $k$ is computed aggregating all such associations, across all labeled examples in $X_{L}$ :

$$
g_{\mathbf{c}}(k)=\frac{\sum_{\mathbf{x} \in X_{L}(k)} f_{\mathbf{x}, \mathbf{c}}(k)}{\sum_{k^{\prime} \in L(t)} \sum_{\mathbf{x} \in X_{L}\left(k^{\prime}\right)} f_{\mathbf{x}, \mathbf{c}}\left(k^{\prime}\right)}, \quad k=1 \ldots L(t)
$$

where $X_{L}(k)$ refers to labeled examples belonging to class $k$. Note that $\sum_{k} g_{\mathbf{c}}(k)=1$.

II. Class informative centroids: If a centroid is associated with only one class $k\left(g_{\mathbf{c}}(k)=1\right)$, only labeled examples of that class select that centroid. At the other extreme, if a 

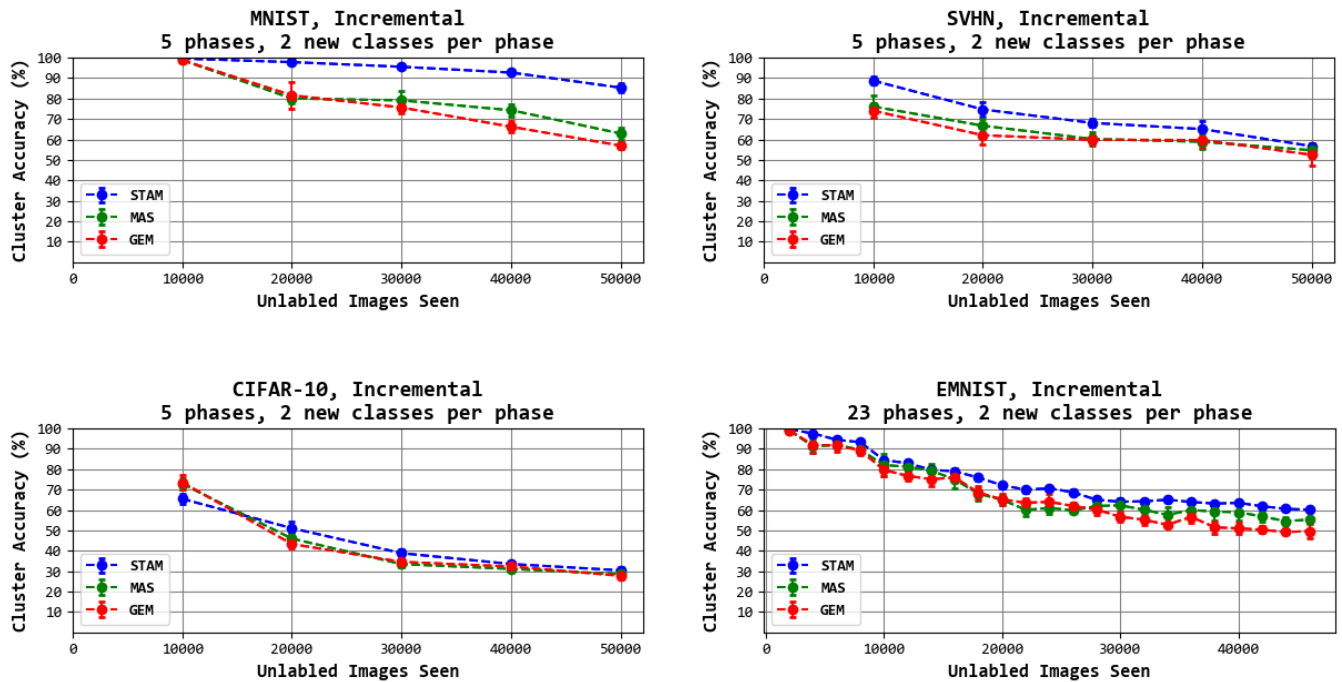

Figure 3: Clustering accuracy for MNIST (left), SVHN (left-center), CIFAR-10 (right-center), and EMNIST (right). The task is expanding clustering for incremental UPL. The number of clusters is equal to 2 times the number of classes in the data stream seen up to that point in time.

centroid is equally likely to be selected by examples of any labeled class, $\left(g_{\mathbf{c}}(k) \approx 1 /|L(t)|\right)$, the selection of that centroid does not provide any significant information for the class of the corresponding input. We identify the centroids that are Class INformative $(C I N)$ as those that are associated with at least one class significantly more than expected by chance. Specifically, a centroid $\mathbf{c}$ is CIN if

$$
\max _{k \in L(t)} g_{\mathbf{c}}(k)>\frac{1}{|L(t)|}+\gamma
$$

where $1 /|L(t)|$ is the chance term and $\gamma$ is the significance term.

III. Classification using a hierarchy of centroids: At test time, we are given an input $\mathbf{x}$ of class $k(\mathbf{x})$ and infer its class as $\hat{k}(\mathbf{x})$. The classification task is a "biased voting" process in which every patch of $\mathbf{x}$, at any layer, votes for a single class as long as that patch selects a CIN centroid.

Specifically, if a patch $\mathrm{x}_{\mathbf{1}, \mathrm{m}}$ of layer $l$ selects a CIN centroid c, then that patch votes $v_{l, m}=\max _{k \in L(t)} g_{\mathbf{c}}(k)$ for the class $k$ that has the highest association with $\mathbf{c}$, and zero for all other classes. If $c$ is not a CIN centroid, the vote of that patch is zero for all classes.

The vote of layer $l$ for class $k$ is the average vote across all patches in layer $l$ (as illustrated in Figure 2):

$$
v_{l}(k)=\frac{\sum_{m \in M_{l}} v_{l, m}}{\left|M_{l}\right|}
$$

where $M_{l}$ is the set of patches in layer $l$. The final inference for input $\mathbf{x}$ is the class with the highest cumulative vote across all layers:

$$
\hat{k}(\mathbf{x})=\arg \max _{k^{\prime}} \sum_{l=1}^{\Lambda} v_{l}(k)
$$

1

\section{Evaluation}

To evaluate the STAM architecture in the UPL context, we consider a data stream in which small groups of classes appear in successive phases, referred to as Incremental UPL. New classes are introduced two at a time in each phase, and they are only seen in that phase. STAM must be able to both recognize new classes when they are first seen in the stream, and to also remember all previously learned classes without catastrophic forgetting. Another evaluation scenario is Uniform UPL, where all classes appear with equal probability throughout the stream - the results for Uniform UPL are shown in SM-G.

We include results on four datasets: MNIST [Lecun et $a l ., 1998$ ], EMNIST (balanced split with 47 classes) [Cohen et al., 2017], SVHN [Netzer et al., 2011] , and CIFAR-10 [Krizhevsky et al., 2014]. For each dataset we utilize the standard training and test splits. We preprocess the images by applying per-patch normalization (instead of image normalization), and SVHN is converted to grayscale. More information about preprocessing can be found in SM-H.

We create the training stream by randomly selecting, with equal probability, $N_{p}$ data examples from the classes seen during each phase. $N_{p}$ is set to $10000,10000,2000$, and 10000 for MNIST, SVHN, EMNIST, and CIFAR-10 respectively. More information about the impact of the stream size can be found in SM-E. In each task, we average results over three different unlabeled data streams. During testing, we select 100 random examples of each class from the test dataset. This process is repeated five times for each training stream (i.e., a total of fifteen results per experiment). The following plots show mean \pm std-dev.

For all datasets, we use a 3-layer STAM hierarchy. In the clustering task, we form the set $\Phi(\mathbf{x})$ considering only Layer-3 patches of the input $\mathbf{x}$. In the classification task, we select a small portion of the training dataset as the labeled examples that are available only to the classifier. The hyperparameter 

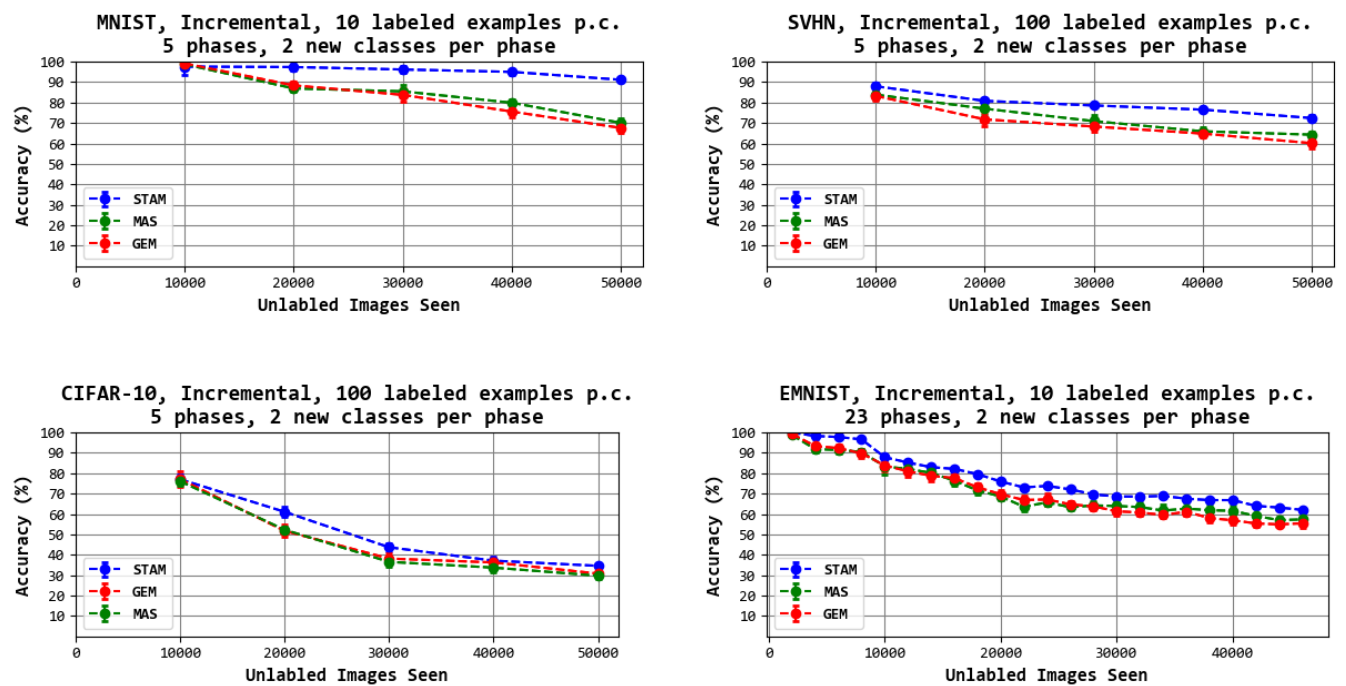

Figure 4: Classification accuracy for MNIST (left), SVHN (center), CIFAR-10 (right-center), and EMNIST (right). The task is expanding classification for incremental UPL, i.e., recognize all classes seen so far. Note that the number of labeled examples is 10 per class (p.c.) for MNIST and EMNIST and 100 per class for SVHN and CIFAR-10.

values are tabulated in SM-A. The robustness of the results with respect to these values is examined in SM-F.

Baseline Methods: We evaluate the STAM architecture comparing its performance to two state-of-the-art baselines for continual learning: GEM and MAS. We emphasize that there are no prior approaches which are directly applicable to UPL. However, we have taken reasonable steps to adapt these two baselines in the UPL setting. Please see SM-B for additional details about our adaptation of GEM and MAS.

Gradient Episodic Memories (GEM) is a recent supervised continual learing model that expects known task boundaries [Lopez-Paz and Ranzato, 2017]. To turn GEM into an unsupervised model, we combined it with a self supervised method for rotation prediction [Gidaris et al., 2018]. Additionally, we allow GEM to know the boundary between successive phases in the data stream. This makes the comparison with STAM somehow unfair, because STAM does not have access to this information. The results show however that STAM performs better even without knowing the temporal boundaries of successive phases.

Memory Aware Synapse (MAS) is another supervised continual learning model that expects known task boundaries [Aljundi et al., 2018]. As in GEM, we combined MAS with a rotation prediction self-supervised task, and provided the model with information about the start of each new phase in the data stream.

To satisfy the stream requirement of UPL, the number of training epochs for both GEM and MAS is set to one. Deep learning methods become weaker in this streaming scenario because they cannot train iteratively over several epochs on the same dataset. For all baselines, the classification task is performed using a $K=1$ Nearest-Neighbor (KNN) classifier - we have experimented with various values of $K$ and other single-pass classifiers, and report only the best performing results here. We have also compared the memory requirement of STAM (storing centroids at STM and LTM) with the memory requirement of the two baselines. The results of that comparison appear in SM-C.

Clustering Task: The results for the clustering task are given in Figure 3. Given that we have the same number of test vectors per class we utilize the purity measure for clustering accuracy. In MNIST, STAM performs consistently better than the two other models, and its accuracy stays almost constant throughout the stream, only dropping slightly in the final phase. In SVHN, STAM performs better than both deep learning baselines with the gap being much smaller in the final phase. In CIFAR-10 and EMNIST, on the other hand, we see similar performance between all three models. Again, we emphasize that STAM is not provided task boundary information while the baselines are and is still able to perform better, significantly in some cases.

Classification Task: We focus on an expanding classification task, meaning that in each phase we need to classify all classes seen so far. The results for the classification task are given in Figure 5. Note that we use only 10 labeled examples per class for MNIST and EMNIST, and 100 examples per class for SVHN and CIFAR-10. We emphasize that the two baselines, GEM and MAS, have access to the temporal boundaries between successive phases, while STAM does not.

As we introduce new classes in the stream, the average accuracy per phase decreases for all methods in each dataset. This is expected, as the task gets more difficult after each phase. In MNIST, STAM performs consistently better than GEM and MAS, and STAM is less vulnerable to catastrophic forgetting. For SVHN, the trend is similar after the first phase but the difference between STAM and both baselines is smaller. With CIFAR-10, we observe that all models including STAM 

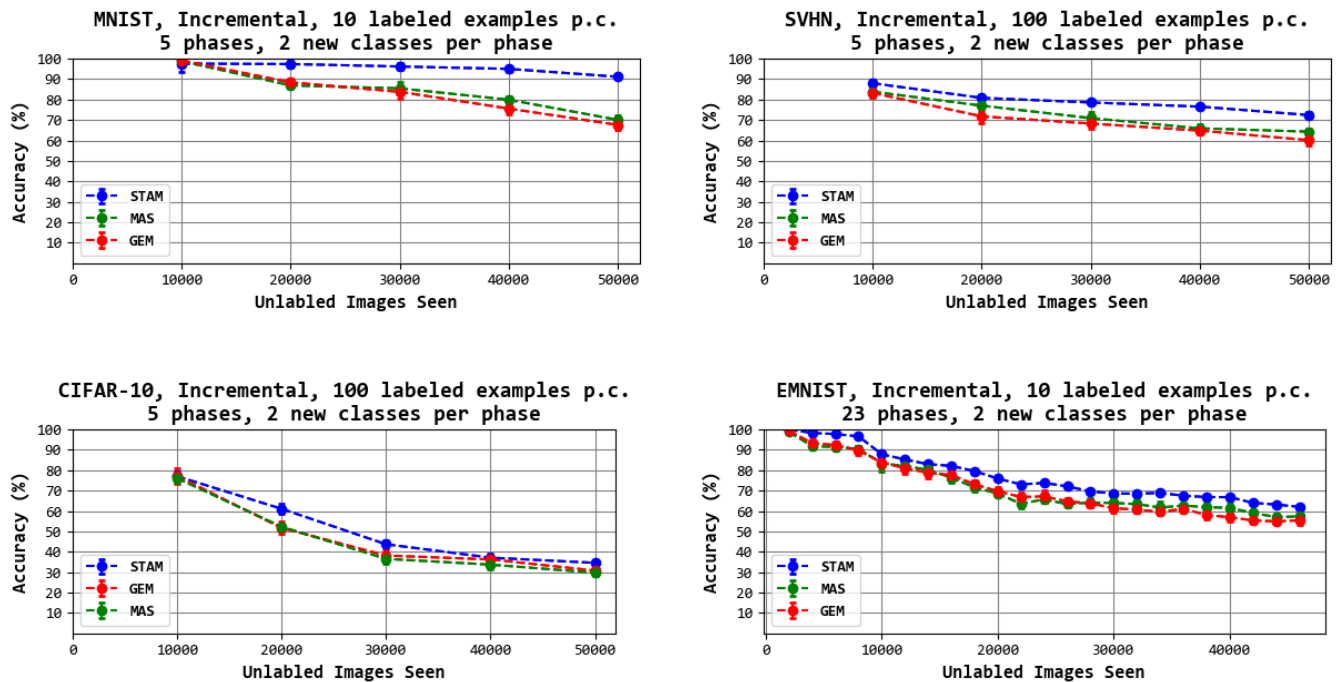

Figure 5: Classification accuracy for MNIST (left), SVHN (center), CIFAR-10 (right-center), and EMNIST (right). The task is expanding classification for incremental UPL, i.e., recognize all classes seen so far. Note that the number of labeled examples is 10 per class (p.c.) for MNIST and EMNIST and 100 per class for SVHN and CIFAR-10.

perform rather poorly - probably due to the low resolution of these images. STAM is still able to maintain comparable accuracy to the baselines with a smaller memory footprint. Finally, in EMNIST, we see a consistently higher accuracy with STAM compared to the two baselines. We would like to emphasize that these baselines are allowed extra information in the form of known tasks boundaries (a label that marks when the class distribution is changing) and STAM is still performs better both on all datasets.

\section{A Closer Look at Incremental UPL}

We take a closer look at STAM performance for incremental UPL in Figure 6. As we introduce new classes to the incremental UPL stream, the architecture recognizes previously learned classes without any major degradation in classification accuracy (left column of Figure 6). The average accuracy per phase is decreasing, which is due to the increasingly difficult expanding classification task. For EMNIST, we only show the average accuracy because there are 47 total classes. In all datasets, we observe that layer-2 and layer-3 (corresponding to the largest two receptive fields) contain the highest fraction of CIN centroids (center column of Figure 6). The ability to recognize new classes is perhaps best visualized in the LTM centroid count (right column of Figure 6). During each phase the LTM count stabilizes until a sharp spike occurs at the start of the next phase when new classes are introduced. This reinforces the claim that the LTM pool of centroids (i) is stable when there are no new classes, and (ii) is able to recognize new classes via novelty detection when they appear.

In the CIFAR-10 experiment, the initial spike of centroids learned is sharp, followed by a gradual and weak increase in the subsequent phases. The per-class accuracy results show that STAM effectively forgets certain classes in subsequent phases (such as classes 2 and 3), suggesting that there is room for improvement in the novelty detection algorithm because the number of created LTM centroids was not sufficiently high.

In the EMNIST experiment, as the number of classes increases towards 47 , we gradually see fewer "spikes" in the LTM centroids for the lower receptive fields, which is expected given the repetition of patterns at that small patch size. However, the highly CIN layers 2 and 3 continue to recognize new classes and create centroids, even when the last few classes are introduced.

Ablation studies: Several STAM ablations are presented in Figure 7. On the left, we remove the LTM capability and only use STM centroids for classification. During the first two phases, there is little (if any) difference in classification accuracy. However, we see a clear dropoff during phases 3-5. This suggests that, without the LTM mechanisms, features from classes that are no longer seen in the stream are forgotten over time, and STAM can only successfully classify classes that have been recently seen. We also investigate the importance of having static LTM centroids rather than dynamic centroids (Fig. 7-middle). Specifically, we replace the static LTM with a dynamic LTM in which the centroids are adjusted with the same learning rate parameter $\alpha$, as in STM. The accuracy suffers drastically because the introduction of new classes "takes over" LTM centroids of previously learned classes, after the latter are removed from the stream. Similar to the removal of LTM, we do not see the effects of "forgetting" until phases $3-5$. Note that the degradation due to a dynamic LTM is less severe than that from removing LTM completely.

Finally, we look at the effects of removing layers from the STAM hierarchy (Fig. 7-right). We see a small drop in accuracy after removing layer 3 , and a large drop in accuracy after also removing layer 2 . The importance of having a deeper hierarchy would be more pronounced in datasets with higher-resolution images or videos, potentially showing multi- 

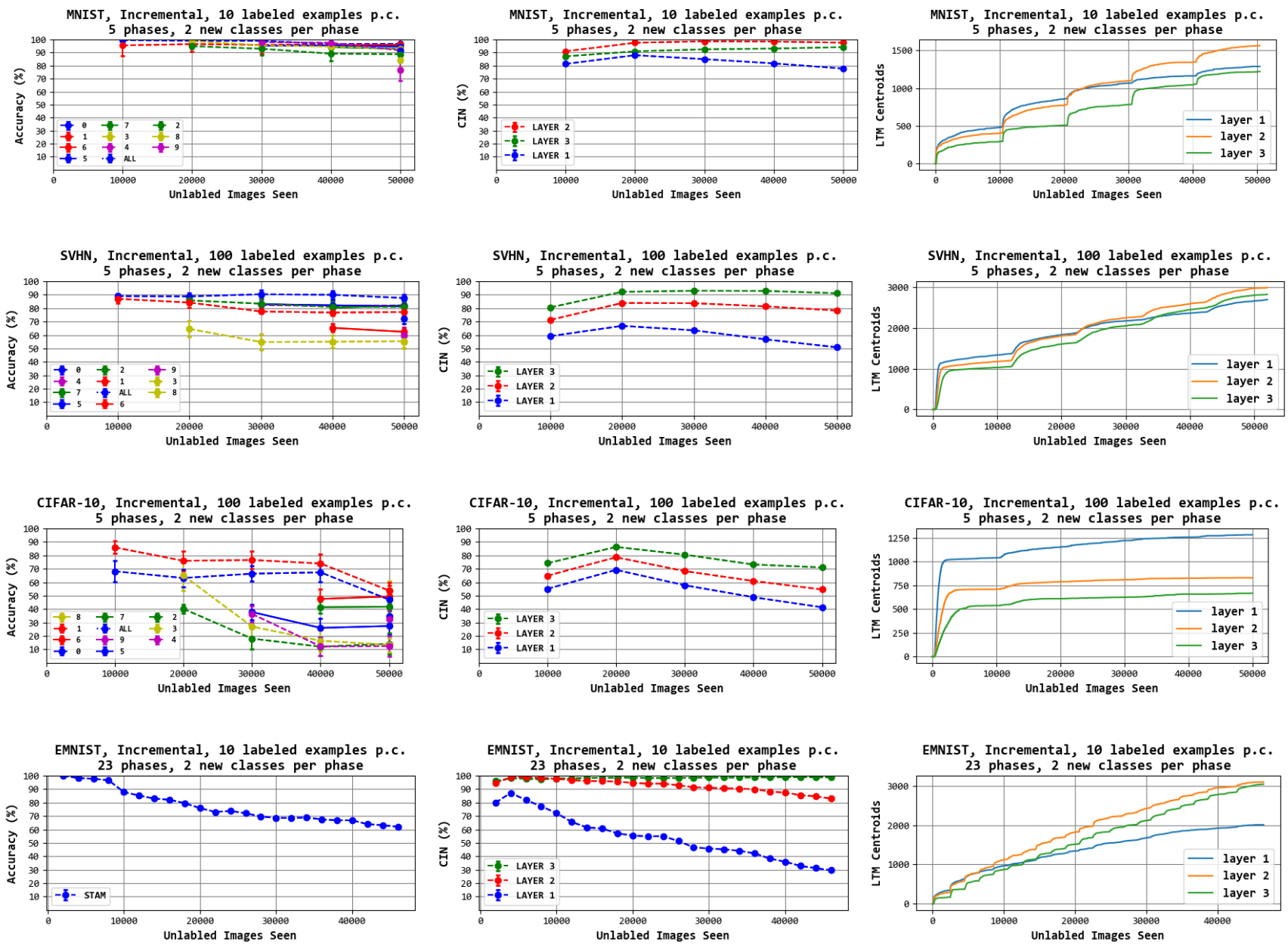

Figure 6: STAM Incremental UPL evaluation for MNIST (row-1), SVHN (row-2), EMNIST (row-3) and CIFAR-10 (row-4). Per-class (p.c.) and average classification accuracy (left); fraction of CIN centroids over time (center); number of LTM centroids over time (right). The task is expanding classification, i.e., recognize all classes seen so far.
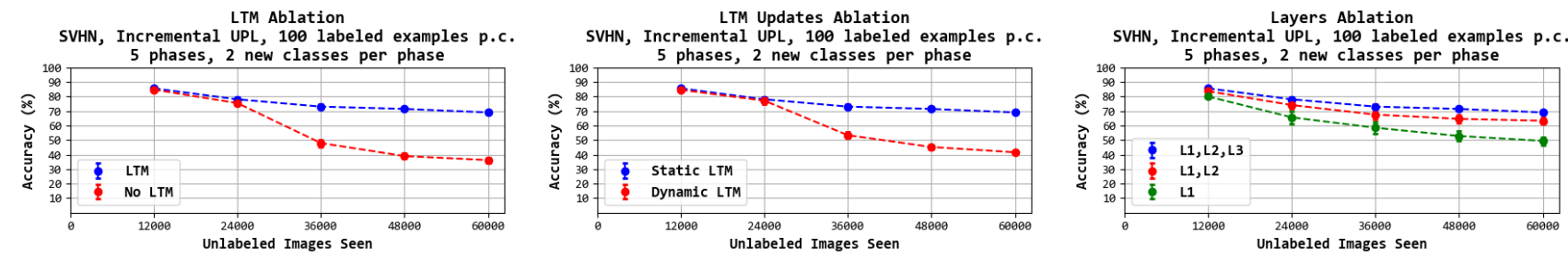

Figure 7: Ablation study: A STAM architecture without LTM (left), a STAM architecture in which the LTM centroids are adjusted with the same learning rate $\alpha$ as in STM (center), and a STAM architecture with removal of layers (right). The number of labeled examples is 100 per class (p.c.).

ple objects in the same frame. In such cases, CIN centroids can appear at any layer, starting from the lowest to the highest.

\section{Related Work}

I: Continual learning: The main difference between most continual learning approaches and STAM is that they are de- signed for supervised learning, and it is not clear how to adapt them for online and unlabeled data streams [Aljundi et al., 2018; Aljundi et al., 2019; Lopez-Paz and Ranzato, 2017].

II. Offline unsupervised learning: These methods require prior information about the number of classes present in a given dataset and iterative training (i.e. data replay) [Bengio 
et al., 2013].

III. Semi-supervised learning (SSL): SSL methods require labeled data during the representation learning stage [Kingma et al., 2014].

IV. Few-shot learning (FSL) and Meta-learning: These methods recognize object classes not seen in the training set with only a single (or handful) of labeled examples [Vanschoren, 2018]. Similar to SSL, FSL methods require labeled data to learn representations.

V. Multi-Task Learning (MTL): Any MTL method that involves separate heads for different tasks is not compatible with UPL because task boundaries are not known a priori in UPL [Ruder, 2017]. MTL methods that require pre-training on a large labeled dataset are also not applicable to UPL.

VI. Online and Progressive Learning: Many earlier methods learn in an online manner, meaning that data is processed in fixed batches and discarded afterwards. These methods are often designed to work with supervised datastreams, stationary streams, or both [Venkatesan and Er, 2016].

VII. Unsupervised Continual Learning: Similar to the UPL problem, CURL [Rao et al., 2019] focuses on continual unsupervised learning from non-stationary data with unknown task boundaries. Like STAM, CURL also includes a mechanism to trigger dynamic capacity expansion as the data distribution changes. However, a major difference is that CURL is not a streaming method - it processes each training example multiple times. We have experimented with CURL but we found that its performance collapses in the UPL setting due to mostly two reasons: the single-pass through the data requirement of UPL, and the fact that we can have more than one new classes per phase. For these reasons, we choose not to compare STAM with CURL because such a comparison would not be fair for the latter.

iLAP [Khare et al., 2021] learns classes incrementally by analyzing changes in performance as new data is introduced it assumes however a single new class at each transition and known class boundaries. [He and $\mathrm{Zhu}, 2021$ ] investigate a similar setting where pseudo-labels are assigned to new data based on cluster assignments but assumes knowledge of the number of classes per task and class boundaries.

VIII. Clustering-based representation learning: Clustering has been used successfully in the past for offline representation learning (e.g., [Coates et al., 2011]). Its effectiveness, however, gradually drops as the input dimensionality increases [Beyer et al., 1999]. In the STAM architecture, we avoid this issue by clustering smaller subvectors (patches) of the input data. If those subvectors are still of high dimensionality, another approach is to reduce the intrinsic dimensionality of the input data at each layer by reconstructing that input using representations (selected centroids) from the previous layer.

IX. Other STAM components: The online clustering component of STAM can be implemented with a rather simple recurrent neural network of excitatory and inhibitory spiking neurons, as shown recently [Pehlevan et al., 2017]. The novelty detection component of STAM is related to the problem of anomaly detection in streaming data [Dasgupta et al., 2018]. Finally, brain-inspired dual-memory systems have been proposed before for memory consolidation (e.g., [Parisi et al., 2018; Shin et al., 2017]).

\section{Discussion}

The STAM architecture aims to address the following desiderata that is often associated with Lifelong Learning:

I. Online learning: STAMs update the learned features with every observed example. There is no separate training stage for specific tasks, and inference can be performed in parallel with learning.

II. Transfer learning: The features learned by the STAM architecture in earlier phases can be also encountered in the data of future tasks (forward transfer). Additionally, new centroids committed to LTM can also be closer to data of earlier tasks (backward transfer).

III. Resistance to catastrophic forgetting: The STMLTM memory hierarchy of the STAM architecture mitigates catastrophic forgetting by committing to "permanent storage" (LTM) features that have been often seen in the data during any time period of the training period.

IV. Expanding learning capacity: The unlimited capacity of LTM allows the system to gradually learn more features as it encounters new classes and tasks. The relatively small size of STM, on the other hand, forces the system to forget features that have not been recalled frequently enough after creation.

V. No direct access to previous experience: STAM only needs to store data centroids in a hierarchy of increasing receptive fields - there is no need to store previous exemplars or to learn a generative model that can produce such examples.

\section{Acknowledgements}

This work is supported by the Lifelong Learning Machines (L2M) program of DARPA/MTO: Cooperative Agreement HR0011-18-2-0019. The authors acknowledge the comments of Zsolt Kira for an earlier version of this work.

\section{References}

[Aljundi et al., 2018] Rahaf Aljundi, Francesca Babiloni, Mohamed Elhoseiny, Marcus Rohrbach, and Tinne Tuytelaars. Memory aware synapses: Learning what (not) to forget. In $E C C V, 2018$.

[Aljundi et al., 2019] Rahaf Aljundi, Klaas Kelchtermans, and Tinne Tuytelaars. Task-free continual learning. In Proceedings of the IEEE/CVF Conference on Computer Vision and Pattern Recognition, pages 11254-11263, 2019.

[Bengio et al., 2013] Yoshua Bengio, Aaron Courville, and Pascal Vincent. Representation learning: A review and new perspectives. IEEE Trans. Pattern Anal. Mach. Intell., 35(8):1798-1828, August 2013.

[Beyer et al., 1999] Kevin S. Beyer, Jonathan Goldstein, Raghu Ramakrishnan, and Uri Shaft. When is "nearest neighbor" meaningful? In Proceedings of the 7th International Conference on Database Theory, ICDT '99, pages 217-235, London, UK, UK, 1999. Springer-Verlag.

[Coates et al., 2011] Adam Coates, Andrew Ng, and Honglak Lee. An analysis of single-layer networks in unsupervised feature learning. In Proceedings of the fourteenth international conference on artificial intelligence and statistics, pages 215-223, 2011. 
[Cohen et al., 2017] Gregory Cohen, Saeed Afshar, Jonathan Tapson, and André van Schaik. EMNIST: an extension of MNIST to handwritten letters. ArXiv, abs/1702.05373, 2017.

[Dasgupta et al., 2018] Sanjoy Dasgupta, Timothy C Sheehan, Charles F Stevens, and Saket Navlakha. A neural data structure for novelty detection. Proceedings of the National Academy of Sciences, 115(51):13093-13098, 2018.

[Gidaris et al., 2018] Spyros Gidaris, Praveer Singh, and Nikos Komodakis. Unsupervised representation learning by predicting image rotations. In International Conference on Learning Representations, 2018.

[Goldstone, 1998] Robert L Goldstone. Perceptual learning. Annual review of psychology, 49(1):585-612, 1998.

[Goodfellow et al., 2013] Ian J Goodfellow, Mehdi Mirza, Da Xiao, Aaron Courville, and Yoshua Bengio. An empirical investigation of catastrophic forgetting in gradient-based neural networks. arXiv preprint arXiv:1312.6211, 2013.

[He and Zhu, 2021] Jiangpeng He and Fengqing Zhu. Unsupervised continual learning via pseudo labels. arXiv preprint arXiv:2104.07164, 2021.

[Hsu et al., 2018] Yen-Chang Hsu, Yen-Cheng Liu, Anita Ramasamy, and Zsolt Kira. Re-evaluating continual learning scenarios: A categorization and case for strong baselines. In NeurIPS Continual learning Workshop, 2018.

[Khare et al., 2021] Shivam Khare, Kun Cao, and James Rehg. Unsupervised class-incremental learning through confusion. arXiv preprint arXiv:2104.04450, 2021.

[Kingma et al., 2014] Diederik P. Kingma, Danilo J. Rezende, Shakir Mohamed, and Max Welling. Semisupervised learning with deep generative models. In Proceedings of the 27th International Conference on Neural Information Processing Systems - Volume 2, NIPS'14, pages 3581-3589, Cambridge, MA, USA, 2014. MIT Press.

[Krizhevsky et al., 2014] Alex Krizhevsky, Vinod Nair, and Geoffrey Hinton. The cifar-10 dataset. online: http://www. cs. toronto. edu/kriz/cifar. html, 55, 2014.

[Kumaran et al., 2016] Dharshan Kumaran, Demis Hassabis, and James L McClelland. What learning systems do intelligent agents need? complementary learning systems theory updated. Trends in cognitive sciences, 20(7):512534, 2016.

[Lecun et al., 1998] Y. Lecun, L. Bottou, Y. Bengio, and P. Haffner. Gradient-based learning applied to document recognition. Proceedings of the IEEE, 86(11):2278-2324, Nov 1998.

[Lopez-Paz and Ranzato, 2017] David Lopez-Paz and Marc'Aurelio Ranzato. Gradient episodic memory for continual learning. In Proceedings of the 31st International Conference on Neural Information Processing Systems, NIPS'17, pages 6470-6479, USA, 2017. Curran Associates Inc.

[Netzer et al., 2011] Yuval Netzer, Tao Wang, Adam Coates, Alessandro Bissacco, Bo Wu, and Andrew Y. Ng. Reading digits in natural images with unsupervised feature learning. In NIPS Workshop on Deep Learning and Unsupervised Feature Learning 2011, 2011.

[Parisi et al., 2018] German I Parisi, Jun Tani, Cornelius Weber, and Stefan Wermter. Lifelong learning of spatiotemporal representations with dual-memory recurrent selforganization. Frontiers in neurorobotics, 12:78, 2018.

[Pehlevan et al., 2017] Cengiz Pehlevan, Alexander Genkin, and Dmitri B Chklovskii. A clustering neural network model of insect olfaction. In 2017 51st Asilomar Conference on Signals, Systems, and Computers, pages 593-600. IEEE, 2017.

[Rao et al., 2019] Dushyant Rao, Francesco Visin, Andrei Rusu, Razvan Pascanu, Yee Whye Teh, and Raia Hadsell. Continual unsupervised representation learning. In Advances in Neural Information Processing Systems 32, pages 7645-7655. Curran Associates, Inc., 2019.

[Rebuffi et al., 2017] Sylvestre-Alvise Rebuffi, Alexander Kolesnikov, Georg Sperl, and Christoph H. Lampert. iCaRL: Incremental classifier and representation learning. In 2017 IEEE Conference on Computer Vision and Pattern Recognition, CVPR'17, pages 5533-5542, 2017.

[Ruder, 2017] Sebastian Ruder. An overview of multitask learning in deep neural networks. arXiv preprint arXiv:1706.05098, 2017.

[Shin et al., 2017] Hanul Shin, Jung Kwon Lee, Jaehong Kim, and Jiwon Kim. Continual learning with deep generative replay. In I. Guyon, U. V. Luxburg, S. Bengio, H. Wallach, R. Fergus, S. Vishwanathan, and R. Garnett, editors, Advances in Neural Information Processing Systems 30, pages 2990-2999. Curran Associates, Inc., 2017.

[Smith et al., 2021] James Smith, Cameron Taylor, Seth Baer, and Constantine Dovrolis. Unsupervised progressive learning and the STAM architecture. arXiv preprint arXiv:1904.02021, 2021.

[Vanschoren, 2018] Joaquin Vanschoren. Meta-learning: A survey. arXiv preprint arXiv:1810.03548, 2018.

[Venkatesan and Er, 2016] Rajasekar Venkatesan and Meng Joo Er. A novel progressive learning technique for multi-class classification. Neurocomput., 207(C):310-321, September 2016.

[Zeno et al., 2018] Chen Zeno, Itay Golan, Elad Hoffer, and Daniel Soudry. Task agnostic continual learning using online variational bayes. arXiv preprint arXiv:1803.10123, 2018. 